Revue internationale P.M.E.

Économie et gestion de la petite et moyenne entreprise

\title{
Les PME à forte croissance et la métaphore du jazz. Comment gérer l'improvisation de façon cohérente
}

\section{Pierre-André Julien}

Volume 14, numéro 3-4, 2001

URI : https://id.erudit.org/iderudit/1008700ar

DOI : https://doi.org/10.7202/1008700ar

Aller au sommaire du numéro

Éditeur(s)

Presses de l’Université du Québec

ISSN

0776-5436 (imprimé)

1918-9699 (numérique)

Découvrir la revue

Citer cet article

Julien, P.-A. (2001). Les PME à forte croissance et la métaphore du jazz. Comment gérer l'improvisation de façon cohérente. Revue internationale P.M.E., 14(3-4), 129-161. https://doi.org/10.7202/1008700ar

\section{Résumé de l'article}

La métaphore de l'orchestre de jazz semble particulièrement appropriée pour analyser le comportement des PME à forte croissance qui doivent continuellement se réorganiser face à ces changements. Nous avons utilisé cette métaphore dans une étude de 52 cas de PME québécoises à forte croissance. Les résultats montrent qu'au-delà de la croissance même du secteur ou des stratégies de fusion ou d'acquisition, le succès de ces firmes relève non seulement du dynamisme de la direction et des relations particulières qu'elles établissent avec les clients, mais avant tout de la capacité de l'organisation à s'adapter au changement et, donc, de leurs pratiques d'autoréorganisation à mesure que la croissance exige plus de ressources et plus de capacité pour innover et relever ainsi les défis du développement des marchés. C'est pourquoi elles recourent fréquemment aux ressources dans l'environnement, notamment à des conseillers privés et publics, et plusieurs d'entre elles entretiennent des contacts particuliers avec des institutions d'enseignement et de R-D, ce qu'on appelle généralement des réseaux à signaux faibles. Ainsi, elles agissent à la manière d'un orchestre de jazz possédant un leader et des joueurs de grande qualité et profitant du passage de collègues étrangers pour improviser de façon harmonieuse et toujours plus loin dans l'invention, charmant et conservant ainsi un public averti.
Ce document est protégé par la loi sur le droit d'auteur. L'utilisation des services d'Érudit (y compris la reproduction) est assujettie à sa politique d'utilisation que vous pouvez consulter en ligne.

https://apropos.erudit.org/fr/usagers/politique-dutilisation/ 


\title{
Les PME à forte croissance et la métaphore du jazz. Comment gérer l'improvisation de façon cohérente
}

\author{
Pierre-André JULIEN \\ Chaire Bombardier en gestion du changement technologique dans les PME \\ Institut de recherche sur les $P M E$ \\ Université du Québec à Trois-Rivières
}

MOTS CLÉS
PME à forte croissance - Improvisation - Métaphore du jazz Organisations apprenantes - Auto-organisation Réseaux à signaux faibles

\begin{abstract}
RÉSUMÉ
La métaphore de l'orchestre de jazz semble particulièrement appropriée pour analyser le comportement des PME à forte croissance qui doivent continuellement se réorganiser face à ces changements. Nous avons utilisé cette métaphore dans une étude de 52 cas de PME québécoises à forte croissance. Les résultats montrent qu'au-delà de la croissance même du secteur ou des stratégies de fusion ou d'acquisition, le succès de ces firmes relève non seulement du dynamisme de la direction et des relations particulières qu'elles établissent avec les clients, mais avant tout de la capacité de l'organisation à s'adapter au changement et, donc, de leurs
\end{abstract}

\section{L'AUTEUR}

Pierre-André Julien détient un doctorat en sciences économiques de l'Université catholique de Louvain et un doctorat honoris causa de l'École nationale polytechnique de Lorraine. Il est titulaire de la chaire Bombardier Produits récréatifs en gestion du changement technologique dans les PME et directeur de la Revue. Présentement, il travaille avant tout sur la gestion de l'information dans les PME et dans les réseaux d'entreprises. Il a publié à ce jour près de 80 articles dans des revues internationales et 19 ouvrages. Courriel: <pierre-andre_julien @uqtr.cas, 
pratiques d'autoréorganisation à mesure que la croissance exige plus de ressources et plus de capacité pour innover et relever ainsi les défis du développement des marchés. C'est pourquoi elles recourent fréquemment aux ressources dans l'environnement, notamment à des conseillers privés et publics, et plusieurs d'entre elles entretiennent des contacts particuliers avec des institutions d'enseignement et de $R-D$, ce qu'on appelle généralement des réseaux à signaux faibles. Ainsi, elles agissent à la manière d'un orchestre de jazz possédant un leader et des joueurs de grande qualité et profitant du passage de collègues étrangers pour improviser de façon harmonieuse et toujours plus loin dans l'invention, charmant et conservant ainsi un public averti.

\begin{abstract}
The jazz band metaphor seems particularly useful in understanding the behaviour of fast growth firms. We used it in case studies of 52 small and medium-sized fastgrowth firms in Québec. Beyond the growth of the sector itself, our results show that the success of fast-growth firms ("gazelles") depends on their ability to respond to their customers' needs, to manage change and to reorganize their organization systematically. Their management is experienced and communicative, and the firms have a very strong market orientation, particularly for exporting. However, as with a very good jazz band, the organization of such firms is particularly complex, decentralized, participative and learning in nature, linked by a dynamic and evolutive culture. And, like the better free jazz bands, the firms make use of resources in the environment, including private and public consultants, to innovate and change and also maintain special contacts with higher educational institutions and $R$ - $D$ centres. Such firms therefore act like free jazz bands by taking advantage of visiting players to improvize with greater harmony, to innovate, and to respond with new knowledge to an audience "in the know".
\end{abstract}

\title{
RESUMEN
}

La metáfora de la orquesta de jazz parece especialmente apropiada para analizar el comportamiento de las PyMEs de fuerte crecimiento que deben reorganizarse constantemente ante esos cambios. Nosotros hemos utilizado esa metáfora en el estudio de 52 casos de PyMEs de Quebec de fuerte crecimiento. Los resultados muestran que, independientemente del crecimiento del propio sector o de estrategias de fusiones o de adquisiciones, el éxito de estas empresas proviene no sólo de la dinámica de la dirección o de la relación que ésta desarrolla con sus clientes, sino sobre todo de la capacidad de la organización para adaptarse a los cambios $y$, por ello, de sus prácticas de autoreorganización a medida que el crecimiento exige más recursos y capacidad para innovar y para superar el desafío del desarrollo de los mercados. Ésta es la causa de que recurran a menudo a los recursos del entorno, especialmente a los asesores públicos y privados. Muchas de estas empresas mantienen contactos particulares con instituciones de enseñanza y de $1+D$, lo que se denomina redes generalmente a señal débil. Las empresas actúan así como lo haría una orquesta de jazz, compuestas de un líder y de intérpretes de gran calidad, beneficiándose del paso de colegas extranjeros para improvisar de modo armonioso y siempre más allá en la invención, atrayendo y conservando así un público exigente.

Revue internationale P.M.E., vol. 14, nos 3-4, 2001 


\section{ZUSAMMENFASSUNG}

Das Jazzorchester als Metapher erscheint besonders geeignet zu sein um das Verhalten der stark wachsenden KMU zu analysieren. Diese müssen sich laufend reorganisieren um die Veränderungen zu verarbeiten. Wir haben diese Metapher angewendet in einer Untersuchung mit 52 stark wachsenden KMU in Québèc.

Die Resultate zeigen, dass neben dem Branchenwachstum, der strategischen Fusionsstrategie, den Akquisitionen, der Erfolg dieser Betriebe nicht nur auf der Dynamik der Unternehmensleitung und den speziellen Kundenbeziehungen beruht. Der Erfolg beruht vor allem auf der Fähigkeit, die Organisation auf die Veränderungen einzurichten. Der Effekt dieser Selbstorganisation erlaubt, mehr Kapazität zu reservieren für Innovationen und um sich den Herausforderungen des Marktes zu stellen.

Aus diesem Grunde beanspruchen die Unternehmen häufig Ressourcen aus der Umwelt. Einerseits von privaten und öffentlichen Beratern und andererseits unterhalten mehrere Unternehmen spezielle Kontakte mit Ausbildungsinstitutionen und Forschungs- und Entwicklungsstätten. Die Unternehmen agieren ähnlich wie ein Jazzorchester, mit einem Leader und Spielern mit grosser Qualität, die vom Kontakt mit ausländischen Kollegen profitieren und in einer harmonischen, innovativen Art improvisieren.

\section{Introduction}

Au cours des dernières années, plusieurs chercheurs, tels Berliner (1994), Brown et Eisenhardt (1998), Choo (1998), Barret (1998) ou Avenier (2000)1 ont utilisé la métaphore de l'orchestre de jazz pour illustrer la capacité de certaines entreprises à gérer l'improvisation dans leur organisation dans des périodes de changement rapide non seulement quant à leurs procédés et leurs produits, mais aussi quant à la réorganisation continuelle de leur personnel pour mieux répondre aux besoins des clients et à l'évolution de l'environnement. Le recours à cette métaphore a été toutefois critiqué au regard des comportements de croissance normale des entreprises (Zack, $2000)^{2}$; mais il peut être utile pour mieux comprendre comment les petites entreprises à forte croissance ${ }^{3}$, les gazelles comme les nomment Birch, Haggerty et Parsons (1997), à la condition évidemment d'en respecter les limites (Ricœur, 1975).

1. Cette dernière auteure étendant aussi la métaphore au petit orchestre de chambre. Voir aussi dans ce cas Arian (1971), cité par Weick, Gilfillan et Keith (1973).

2. À noter que M.H. Zack, chercheur au Collège d'administration des affaires de l'Université Northeastern aux États-Unis, est lui-même un jazzman averti.

3. Ce recours provient d'une discussion à partir de mon idée d'improvisation avec notre collègue Philippe Mustar, responsable de l'étude internationale, lors de la présentation du rapport à l'OCDE à Bologne, le 12 juin 2000. Par la suite, un de nos doctorants, Ivan Vaghely, nous a signalé que cette analogie avait été utilisée par d'autres auteurs, tels Weick, Gilfillan et Keith (1973). De fil en aiguille, nous avons trouvé de plus en plus d'auteurs recourant à cette métaphore, y compris dans le numéro spécial de Organization Science, vol. 9, n 5, 1998.

Revue internationale P.M.E., vol. 14, nos 3-4, 2001 
Pour comprendre cette métaphore, il importe de rappeler les six règles du jazz d'improvisation, bien résumées par Sabatella (1992) et Hatch (1997), comme l'ont noté Brown et Eisenhardt (1998). Ainsi, dans ces orchestres :

1. chaque joueur doit reconnaître le leader (le soliste), quelle que soit la position et le moment où l'on joue;

2. le soliste doit entendre et comprendre le jeu des autres membres de l'orchestre;

3. tous les joueurs doivent connaître les règles du jeu et comment et quand on peut les contourner ou les ignorer;

4. on doit comprendre si le jeu se fait en groupe (en changeant ou éliminant les structures) ou individuellement (par exemple, jouer ou souffler plus fort);

5. on doit prévoir les sorties ou même les déraillages et être capables de revenir rapidement dans le rythme;

6. on ne doit pas jouer le même solo à n'en plus finir et plutôt pratiquer de nouvelles approches et de styles, même dans les pièces familières.

À ces six règles, nous allons en ajouter une septième qui nous servira par la suite, soit :

7. inviter régulièrement des joueurs étrangers pour échanger de nouvelles approches, de nouvelles techniques de jeu, de nouveaux styles ou de nouveaux airs.

Ces règles peuvent valoir pour les gazelles en les adaptant. Elles expliquent 1) la capacité de l'organisation à reconnaître le leadership de la direction et à partager la stratégie, 2) la volonté de cette direction de bien comprendre le comportement du personnel et de l'organisation, 3) le partage d'une culture commune et de règles ou de normes à instaurer graduellement, 4) une bonne compréhension et un bon support de l'organisation lorsque les employés ou les équipes prennent des initiatives pour faire avancer l'entreprise, 5) la capacité à corriger le tir et à apprendre des erreurs, 6) l'habileté à innover tant dans les procédés et les produits que dans les techniques et méthodes et, enfin, 7) le recours systématique à des consultants externes et à des réseaux à signaux faibles.

En d'autres mots, ces gazelles développent une capacité particulière à faire face au changement, à gérer le désordre (Chanlat et Dufour, 1985 ; Schmitt, 1999) produit par la forte croissance et à utiliser les ressources externes pour prendre du recul par rapport au quotidien afin de donner de la cohérence et une orientation à l'improvisation de façon à répondre aux besoins actuels et prochains de la clientèle. Leurs pratiques exemplaires peuvent être résumées de la manière suivante : 
1. elles formalisent les routines, c'est-à-dire qu'elles rendent les employés responsables des tâches journalières par diverses pratiques opérationnelles relativement formelles ${ }^{4}$, dont plusieurs informatisées ${ }^{5}$;

2. elles organisent les semi-routines au plan interne en rendant des cadres et des groupes d'employés responsables de changements mineurs ${ }^{6}$ et au plan externe en établissant des liens avec différentes ressources pour obtenir de l'information riche;

3. cette formalisation et cette organisation permettent de libérer la direction pour mieux voir venir le changement et ainsi gérer ce désordre, d'une part, en orientant l'organisation notamment par une planification flexible et en stimulant les troupes par un partage des défis et le développement d'une culture forte d'entreprise et, d'autre part, en faisant le suivi des clients les plus importants et en recherchant de nouveaux clients, pour saisir ainsi les nouvelles occasions d'affaire et prendre les décisions clés permettant de soutenir la croissance.

Nous utiliserons cette métaphore dans l'application de l'étude internationale sur les PME à forte croissance ${ }^{7}$ et pour rappeler leur rôle très important dans la multiplication des emplois malgré leur faible importance en nombre ${ }^{8}$. Nous discuterons d'abord de la méthodologie suivie. À la section suivante, nous présenterons les résultats généraux de cette recherche. À la section 3, nous nous arrêterons aux variables qui discriminent le mieux la forte croissance. À la fin, nous reviendrons sur les points les plus marquants de cette analyse en faisant à nouveau le lien avec la métaphore du jazz.

4. À titre d'exemple, voir les pratiques à base de principes fort simples du poka yoke japonais pour favoriser l'auto-entretien préventif (de base) par les opérateurs des machines, à partir d'instructions de couleur selon les périodes d'entretien.

5. Dans certaines entreprises de notre enquête, les employés étaient directement capables de connaître quotidiennement les coûts engagés et leur production de façon à pouvoir se réajuster rapidement lorsque ces chiffres dépassaient les prévisions.

6. Par exemple, faire gérer un budget d'investissements minimal (entre $5000 \$$ et $10000 \$$ par année) par ateliers ou groupes d'employés, comme c'est le cas de quelques firmes de notre échantillon.

7. Soit les PME ayant entre 10 et 500 employés ( 20 et 500 employés pour l'étude internationale) au début de la période et dont ce nombre avait au moins doublé au cours des dernières années. Au Québec, ce nombre d'employés avait augmenté en moyenne de 2,7 fois entre 1990 et 1996. À noter que cette forte croissance dans une entreprise peut ne s'observer que sur une période limitée et cela ne veut pas nécessairement dire qu'elle va se poursuivre dans les années futures, comme l'ont d'ailleurs fait remarquer Baldwin, Chandler et Papailiadis (1994).

8. Pour le Québec, ces firmes comptaient pour $8 \%$ des PME mais étaient responsables de $41 \%$ des nouveaux emplois durant la période de 1990 à 1996.

Revue internationale P.M.E., vol. 14, $\mathrm{n}^{\text {os }} 3-4,2001$ 


\section{La méthodologie}

Pour la deuxième phase de l'étude internationale, comme nous l'avons mentionné dans l'éditorial de ce numéro thématique, les chercheurs ont utilisé un questionnaire postal, auprès d'un échantillon aléatoire, qui se limitait à obtenir diverses informations sur la trajectoire de croissance des entreprises (linéaire ou par étapes) et les raisons majeures de celle-ci (croissance interne, par fusion ou acquisition), sur l'évolution de leurs relations avec les marchés nationaux ou internationaux, sur l'importance et l'impact des alliances ou autres formes de partenariats ayant favorisé la croissance, sur les principaux éléments d'évolution de l'organisation et de ses compétences et sur le rôle de l'intervention des pouvoirs publics dans cette croissance. Pour notre part, nous avons voulu aller plus loin en incluant d'autres variables touchant la personnalité et le dynamisme de l'entrepreneur au cœur de l'entreprise, de même que des facteurs environnementaux favorables ou défavorables à la croissance ${ }^{9}$, afin de mieux comprendre leurs comportements d'improvisation et leurs recours aux ressources externes. Nous avons utilisé une méthode qui s'apparente à l'étude de cas ${ }^{10}$ auprès de $52 \mathrm{PME}$ provenant d'une liste de $282 \mathrm{PME}$ à forte croissance issue de l'analyse effectuée lors de la première phase. Cette méthode permet, d'une part, de s'assurer d'avoir le bon interlocuteur qui répondra aux questions et de réagir à ses interrogations en lui apportant les précisions nécessaires et, d'autre part, de laisser un grand nombre de questions ouvertes afin d'obtenir toutes sortes d'informations non prévues à mesure que les entrevues se déroulent.

Les entreprises ont été choisies dans sept régions du Québec, tant celle métropolitaine que des régions intermédiaires et périphériques ${ }^{11}$. Des entreprises qui ont

9. Signalons que notre étude diffère des nombreuses études sur les stades de vie des entreprises, études issues des premiers travaux de Penrose (1959); (voir, par exemple, une telle étude récente menée par Divay, 1999): elle ne porte pas sur une très longue période et présente des trajectoires beaucoup plus complexes que ces études relativement déterministes; d'autant plus que ces études sur les stades de vie ne tiennent pas compte du plus grand nombre de petites entreprises qui demeurent petites (Davidsson, 1989; Julien, 2000).

10. Huberman et Miles (1991) admettent une série de questionnaires s'ajoutant à des entrevues complexes comme des études de cas, alors que Yin (1989) est beaucoup plus strict sur ce point, en ne considérant comme telle que les études qui comprennent des rencontres avec plusieurs membres d'une organisation et ce, en plusieurs étapes.

11. Dans deux régions, comme le nombre d'entreprises à forte croissance tiré de la banque était insuffisant, nous avons dû faire appel à des intervenants régionaux; de même, comme aucune banque touchant les services aux entreprises ne donnait l'évolution des emplois, nous avons utilisé le bouche à oreille pour trouver celles qui avaient fortement crû dans les dernières années. 
répondu, 46 évoluaient dans le secteur manufacturier ${ }^{12}$ et six dans celui des services aux entreprises ${ }^{13}$. À l'annexe I, nous donnons la liste de ces entreprises selon ces différentes branches industrielles, la variation de l'emploi et du chiffre d'affaires et soumettons quelques observations sur les points majeurs qualifiant la croissance. Signalons que la plupart de ces entreprises (sauf trois) évoluent dans les industries à technologie moyenne, ce qui indique que cette croissance ne peut s'expliquer par leur présence dans les nouvelles industries.

Nous avons utilisé quatre types d'outils dont deux questionnaires. Le premier comprenait des questions qu'un membre de la direction (le plus souvent un cadre autre que le PDG) remplissait seul et qui portaient sur des éléments factuels (l'âge de l'entreprise, le nombre et le type d'employés, la localisation, le type de produits et de services offerts, les technologies utilisées, etc.), soit 36 questions plus ou moins détaillées et fermées dont cinq ordinales. Le second était composé de questions ouvertes et devait être rempli par l'interviewer lors d'une entrevue avec le propriétairedirigeant; il s'arrêtait aux principales caractéristiques du propriétaire-dirigeant (son expérience, sa formation, ses intérêts selon différentes tâches, etc.), sur ses objectifs personnels et ses intentions, sur les origines de l'entreprise et de sa direction et sur les facteurs de succès de l'entreprise. Ce dernier questionnaire était suivi d'une longue entrevue d'environ deux heures et, dans plusieurs cas, d'une visite de l'entreprise. Cette entrevue était réalisée à l'aide d'une grille portant sur les activités et les pratiques de gestion, notamment la stratégie générale et les stratégies fonctionnelles privilégiées, sur l'histoire de l'entreprise, sur les motivations de sa direction, sur le type d'organisation et les niveaux de participation de celle-ci et, enfin, sur les facteurs favorables et les freins ou obstacles à la croissance, facteurs soit événementiels, soit informationnels affectant la décision, comme le recommande McPhee (1990). Enfin, nous avons interrogé neuf intervenants extérieurs (fonctionnaires régionaux, consultants, financiers, etc.) ayant travaillé avec plusieurs de ces firmes pour confronter leurs analyses avec les impressions ou les faits retenus par les propriétaires-dirigeants.

Ajoutons que deux des entreprises de l'échantillon sont membres depuis plusieurs années de la chaire Bombardier ${ }^{14}$ et ont profité des interventions lourdes (de cinq mois) pour implanter la production à valeur ajoutée et développer un plan

12. Aliments : trois entreprises; produits de plastique ou de caoutchouc : cinq; vêtement: un ; meubles : quatre ; produits du bois : deux ; produits métalliques : six ; produits électriques, électroniques et de communications : cinq; matériel de transport: quatre; machinerie et équipements : cinq; produits chimiques et pharmaceutiques : quatre; produits du pétrole : un; divers : six.

13. Deux bureaux d'ingénieurs, deux entreprises en informatique, un bureau comptable et deux entreprises de camionnage.

14. La Chaire est associée depuis 1994 à la grande entreprise Bombardier Produits récréatifs. Elle a pour objectif de créer une entreprise-réseau avec ses sous-traitants et ainsi

Revue internationale P.M.E., vol. 14, nos 3-4, 2001 
d'amélioration continue; ce sont des PME à forte croissance qui s'ajoutent à 16 autres gazelles membres de la Chaire et qui nous ont permis d'ajouter aux résultats de cette recherche internationale toutes sortes de subtilités sur les comportements de ces entreprises pouvant expliquer leurs succès (Jacob, Julien et Raymond, 1997).

Pour l'analyse des questionnaires, nous avons utilisé le logiciel SAS qui a permis d'obtenir différentes données descriptives et de réaliser des tests statistiques permettant d'établir divers types de corrélations pour faire ressortir les variables les plus discriminantes. Nous avons aussi tenu compte, pour les questions semiouvertes, de l'ordre d'importance donné par les répondants, notamment avec les questions ordinales.

L'analyse des entrevues avec les propriétaires-dirigeants a été réalisée en trois temps. D'une part, une fiche synthèse a été préparée pour chaque entreprise à partir des enregistrements de celles-ci ; un historique des trajectoires de croissance a ensuite été tracé (voir un exemple à l'annexe II); de plus, les variables ont été compilées et regroupées par la suite selon différents sous-thèmes. D'autre part, un ordre d'importance a été donné à plusieurs des variables qui n'avaient pas déjà été ordonnées par les répondants selon qu'elles favorisaient plus la croissance ou selon qu'elles limitaient, de moins à plus, celle-ci (Morse, 1994). Enfin, cet ordonnancement a été corrigé ou nuancé à partir d'une analyse de la logique de l'historique, des entrevues complémentaires avec les intervenants et, au besoin, de quelques appels auprès des propriétaires-dirigeants pour confirmer ou infirmer certaines conclusions (Wolfe et Jackson, 1987) ; il a été ensuite utilisé dans diverses analyses statistiques.

Les principales caractéristiques de ces firmes, qu'on peut voir en partie au tableau 1 , sont les suivantes :

1. Des trajectoires de croissance différenciées. Ces trajectoires de croissance sont variées. Ainsi, on trouve trois firmes dont le taux annuel de croissance moyenne de l'emploi entre 1990 et $1998^{15}$ a dépassé $100 \%$ et neuf dont ce taux était entre $50 \%$ et $100 \%$, plus une firme dont le chiffre d'affaires a dépassé $50 \%$. On trouve aussi 19 entreprises qui ont crû annuellement entre $20 \%$ et $50 \%$. Il reste 20 entreprises dont la croissance des emplois a été inférieure à $20 \%$, dont trois avec une croissance des emplois plus ou moins nulle; toutefois, la croissance du chiffre d'affaires pour deux

d'engendrer une synergie pour accélérer l'innovation et consolider les capacités concurrentielles de cette firme et de ses sous-traitants. Actuellement, 29 entreprises soustraitantes cotisent à la Chaire et profitent en retour d'interventions lourdes et de diverses recherches-intervention et de formation pour devenir des entreprises de classe mondiale.

15. Pour quatre firmes, nous n'avons pu avoir l'information pour toute cette période, à cause d'une direction relativement nouvelle ou de la création de l'entreprise après 1990. 
de ces dernières a été d'environ $20 \%$ par année ${ }^{16}$. On peut voir, de plus, à l'annexe I, que 20 entreprises ont connu un rythme de croissance du chiffre d'affaires très supérieur à celle des emplois (de 1,75 fois à plus de 3 fois plus) alors que ce fut le contraire pour cinq entreprises (de 1,66 fois à plus de 3 fois). De plus, 17 firmes ont crû à peu près au même rythme tant au plan des emplois qu'à celui du chiffre d'affaires. Pour les 10 dernières entreprises, des données manquaient pour faire cette comparaison. On peut ainsi distinguer trois types de trajectoires, soit une croissance relativement continue (24 firmes, dont 5 à croissance continue rapide), une croissance accélérée après un seuil (16 firmes, dont 9 à croissance très accélérée) et enfin, une croissance fortement irrégulière (4 firmes, avec des hausses et des baisses fortes).

2. Une taille moyenne (98 employés, avec un minimum de 16 et un maximum de 531) dont environ le tiers dans chaque classe de taille sauf pour les plus grandes et une structure assez complexe sur le plan du partage des tâches fonctionnelles. Elles ont en moyenne 6,7 cadres dont 3,6 ingénieurs et une équipe de vente de 5,9 vendeurs en moyenne. Le personnel de bureau comprend 6,3 employés et celui de la production, 75 employés. Évidemment, le nombre de niveaux hiérarchiques augmente avec le nombre d'employés : la moyenne se situe autour de trois niveaux ; mais 10 entreprises ont cinq niveaux et plus. Quant à la direction de l'entreprise, l'entrepreneur est âgé en moyenne de 47 ans et dirige son entreprise depuis environ 13 ans.

TABLEAU 1

Quelques caractéristiques de l'échantillon (49 entreprises)

\begin{tabular}{lcccc}
\hline Variables & & & & \\
\hline Propriété & Indépendante & Filiale & Siège social & \\
\hline Taille & $37(76 \%)$ & $5(10 \%)$ & $7(14 \%)$ & \\
& 11 à 50 & 51 à 100 & 101 à 200 & 201 et plus \\
& employés & employés & employés & 4 (8\%) \\
\hline Nombre de niveaux hiérarchiques & 2 niveaux ou moins & 3 niveaux & 4 niveaux & 5 niveaux et plus \\
& $12(26 \%)$ & $11(24 \%)$ & $16(35 \%)$ & $10(22 \%)$ \\
\hline Âge moyen de la direction & Moy. 46,8 ans & min. 30 ans & max. 55 & \\
\hline Ancienneté de la direction & Moy. 13,3 ans & min. 2 ans & max. 30 ans & \\
\hline
\end{tabular}

16. Rappelons que le choix des entreprises provenait en bonne partie de la première étape portant sur les années 1990 à 1996, alors que l'enquête incluait 1997 et 1998 ; ce qui peut expliquer un certain ralentissement observé chez quelques entreprises, que nous avons tout de même conservées pour connaître les causes de ce ralentissement et pour faire des comparaisons avec les caractéristiques des autres entreprises. 


\section{Les caractéristiques communes aux PME à forte croissance}

Pour présenter les résultats communs à toutes les entreprises, nous nous arrêterons d'abord aux deux éléments les plus connus expliquant le succès des PME, soit la qualité de la direction (Hambrick et Croizer, 1985; Davidsson, 1989; Julien et Marchesnay, 1997) et une très grande proximité des entreprises avec leur marché ou, mieux, leur clientèle (Siegel, Siegel et MacMillan, 1993; Baldwin, Chandler et Papailiadis, 1994 ; Kim et Mauborgne, 1997 ; Barringer, Jones et Lewis, 1998). Mais la métaphore du jazz s'applique surtout à deux autres groupes de variables, soit des pratiques organisationnelles complexes et participatives et des liens particuliers avec des ressources externes dynamiques. Nous terminerons cette section en distinguant les principaux facteurs favorisant la croissance ou la freinant et présenterons les variables qui expliquent les plus fortes croissances qu'elles ont connues.

\subsection{Un leadership qui incite l'organisation à bien répondre aux besoins des clients}

Le premier rôle d'un leader est d'entraîner ses troupes dans le même sens et donc de donner de la cohérence à l'improvisation pour mieux s'adapter au changement continuel, comme dans le cas du jazz libre. Ce leadership est lié à la perception qu'ont les cadres et les employés de l'expérience de leur « chef », de sa compétence (sa crédibilité ${ }^{17}$ ) et de sa capacité à entraîner le personnel pour pouvoir relever le défi du changement que pose la forte croissance, comme l'ont noté Barth (1999) et l'étude de Deloitte \& Touche (1999).

Dans ces firmes, l'entrepreneur est en général plus instruit que la moyenne des dirigeants de PME, puisque $57 \%$ des dirigeants possèdent au moins un diplôme universitaire et $22 \%$ un diplôme collégial ${ }^{18}$. Seuls $17 \%$ n'ont qu'un diplôme secondaire et $4 \%$ n'ont fréquenté l'école qu'au primaire. Leur champ de spécialisation se concentre en sciences de la gestion (au total, 46 \% des dirigeants ont un diplôme en administration, en finance ou en marketing) et en ingénierie (25\%); les autres ont différentes formations techniques (16\%), formation en droit (4\%) ou autres spécialisations $(9 \%)$. Ce sont, de plus, des dirigeants expérimentés, puisqu'ils sont

17. Weick, Gilfillan et Keith (1973) ont mesuré l'effet de la crédibilité dont jouissent les grands compositeurs de jazz sur les petits orchestres de jazz, montrant qu'elle joue un rôle majeur sur leur performance.

18. Soit le diplôme le plus important obtenu avant leur arrivée à la tête de l'entreprise. Notons que la deuxième année collégiale au Québec correspond à la première année universitaire dans la plupart des pays européens.

Revue internationale P.M.E., vol. 14, $\mathrm{n}^{\text {os }} 3-4,2001$ 
à la tête de leur entreprise depuis 13 ans en moyenne, comme nous l'avons déjà mentionné ; mais surtout, $59 \%$ d'entre eux travaillaient dans le même secteur avant d'accéder à cette direction, donnant ainsi une expérience moyenne de 23,6 années dans le secteur. De même, $67 \%$ d'entre eux provenaient d'une famille proche ou très proche entrepreneuriale, ce qui leur a probablement permis d'acquérir très tôt une certaine expérience des affaires et, avant tout, de comprendre ce qu'est un réseau d'entreprises.

Ces dirigeants cherchent régulièrement à améliorer leur compétence et même à reconnaître ses limites avec la volonté de les dépasser. Plus des deux tiers ont reçu de la formation ces dernières années, notamment, par ordre d'importance, en gestion des ressources humaines, en marketing, en exportation ou en technique de gestion. Cette formation représentait entre 31 et 70 heures par année ${ }^{19}$.

Ajoutons que leurs motivations sont intéressantes à distinguer. Le premier objectif de ces dirigeants est la réalisation de profits, ce à quoi on devait s'attendre. Mais la deuxième motivation est plus personnelle, à savoir faire un travail dans lequel on se sent bien, ce que reprend Avenier (2000) en parlant des orchestres de chambre. La troisième est le défi que pose la gestion d'une entreprise en forte croissance, défi souvent relevé à plusieurs comme on l'a vu. Ainsi, le principal facteur de réussite pour eux est une organisation motivée et capable de bien répondre aux besoins des clients comme on le verra plus loin.

Pour ces facteurs de succès, si on les analyse par thèmes (comme on peut le voir en colonne au tableau 2), on trouve d'abord les facteurs touchant la qualité des relations avec les clients qui se distinguent par rapport à trois autres facteurs dont l'importance est relativement semblable (la qualité de la gestion générale, l'importance du personnel et la gestion financière). Les autres facteurs sont encore moins importants.

Mais en examinant de plus près ces thèmes, on relève des différences importantes. En effet, le premier facteur spécifique est la motivation du personnel : $53 \%$ des firmes l'ont cité et $33 \%$ l'ont rangé parmi les cinq facteurs les plus importants. Ce facteur est suivi de trois autres facteurs importants, soit la qualité du service à la clientèle (45\% l'ont cité dont $20 \%$ parmi les cinq premiers), le fait de faire des profits (42\% et $40 \%)$ et finalement, l'augmentation de la part de marché, l'amélioration continue des produits pour répondre aux besoins spécifiques de la clientèle et le fait de devancer les compétiteurs en matière de services (32\% dans tous les cas).

19. Par comparaison, un cours universitaire au Québec comprend environ 45 heures.

Revue internationale P.M.E., vol. 14, nºs 3-4, 2001 


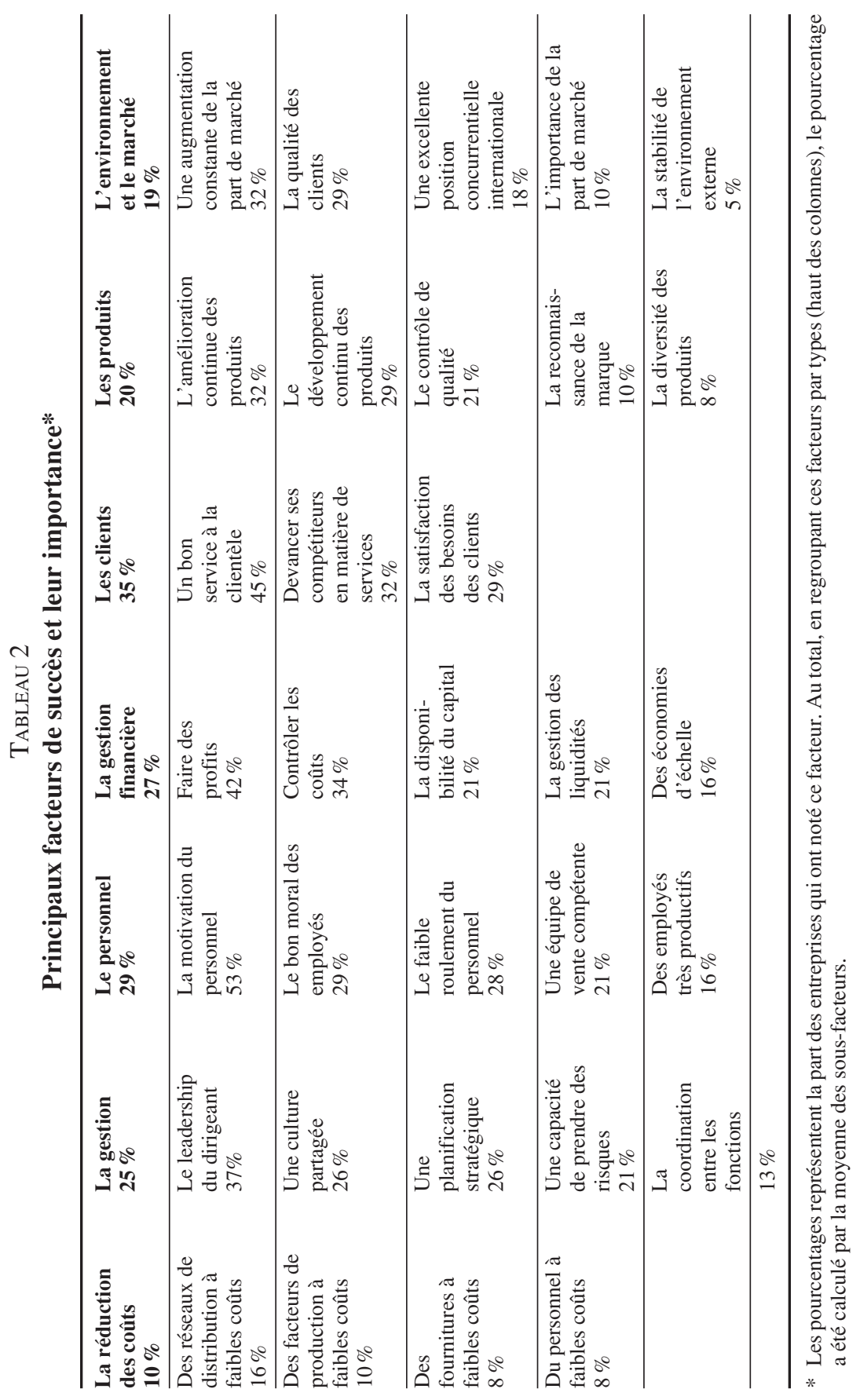

Revue internationale P.M.E., vol. 14, $\mathrm{n}^{\text {os }} 3-4,2001$ 
Deux autres facteurs ressortent en ce qui concerne la direction, soit le leadership du dirigeant $(37 \%)$ et le partage d'une culture d'entreprise par tous $(26 \%)$ aidant fortement à la cohérence de l'organisation, ce qui nous ramène à la motivation du personnel et, par conséquent, à une culture organisationnelle forte relevée notamment par Dyke, Fisher et Reuber (1992) et Kim et Mauborgne (1997). On retrouve cette culture fortement partagée dans l'orchestre de jazz (Barret et Peplowski, 1998).

Cette importance de la motivation du personnel relève de la qualité du leadership et ainsi de la communication entre le PDG et le personnel. Au tableau 2, on peut voir aussi que ce leadership est le quatrième facteur de succès (sur 32) par ordre d'importance. Ce leadership repose sur une culture partagée, une certaine planification et une capacité à prendre des risques.

Le leadership passe aussi par une communication fréquente avec les cadres (avec les joueurs), le plus souvent à travers des rencontres formelles (dans $60 \%$ des cas), des comités de direction et de coordination (52\%); mais on trouve aussi toutes sortes de rencontres informelles. Par exemple, un dirigeant réunit tous les vendredis soir à la brasserie du coin son équipe de direction, soit pour un souper, soit pour la soirée, pour discuter de différentes activités et problèmes de la semaine.

La stratégie évoquée par la direction en est essentiellement une de différenciation (un beat particulier), le plus souvent proactive, sinon active ; on aurait peutêtre pu s'attendre à d'autres stratégies. Il est vrai que Sandberg et Hoffer (1987) et Steiner et Solem (1988) avaient déjà souligné l'importance d'une telle stratégie pour soutenir la forte performance des petites entreprises. Toutes les firmes interrogées ont cette stratégie de différenciation, même si un quart y ajoute des éléments de réduction des coûts et un tiers des éléments de différenciation par une position de niche. Le tableau 3 résume la position des firmes selon ces stratégies. Ainsi, on peut voir que la forte croissance est essentiellement basée sur la volonté d'offrir des produits différents à une clientèle cible.

TABLEAU 3

Proportion des entreprises selon le type de stratégie et leur position en matière d'actions face à l'environnement tout au long de la croissance

\begin{tabular}{lcccccc}
\hline Types de stratégie & $\%$ & \multicolumn{3}{c}{ Position des entreprises face à l'environnement (part de celles-ci en \%) } \\
\hline & \multicolumn{7}{c}{$\begin{array}{c}\text { Proactive } \\
\text { et active }\end{array}$} & Proactive & Active & Réactive & $\begin{array}{c}\text { Proactive } \\
\text { et réactive }\end{array}$ & $\begin{array}{c}\text { Réactive } \\
\text { et active }\end{array}$ \\
\hline Différenciation & 44 & $25 \%$ & $13 \%$ & $6 \%$ & $6 \%$ \\
Différenciation et coûts & 25 & $13 \%$ & $6 \%$ & $6 \%$ & $6 \%$ \\
Différenciation et niche & 31 & $6 \%$ & $19 \%$ & $6 \%$ & & \\
\hline & 100 & & & & & \\
\hline
\end{tabular}

Revue internationale P.M.E., vol. 14, $\mathrm{n}^{\text {os }}$ 3-4, 2001 
Nos analyses statistiques révèlent que parmi les variables qui concernent la direction de l'entreprise, celles corrélées à une plus forte croissance sont le niveau de formation du PDG, son origine familiale entrepreneuriale et sa volonté de poursuivre les objectifs de faire des profits et de relever un défi.

\subsection{Une forte proximité avec la clientèle}

Le type de stratégie nous amène à examiner les relations étroites qu'établissent ces entreprises avec leur marché comme l'orchestre de jazz le fait avec son public acquis. Cette proximité relève de cinq éléments, soit 1) le type, 2) la forme de relations avec les clients, 3) le type de promotion, 4) la capacité de répondre à leurs besoins par l'innovation, avec comme résultat, 5) un contrôle assez important du marché cible par ces firmes à forte croissance.

Cette stratégie de différenciation s'explique, dans plusieurs cas, par une production sur mesure et un système de communication direct avec la clientèle (dans $76 \%$ des cas). Cette proximité particulière des entreprises à forte croissance avait déjà été relevée par Siegel, Siegel et MacMillan (1993). Elle permet de recevoir très rapidement les impulsions du marché pour garder l'avance sur les compétiteurs (Minguzzi et Passaro, 2000) et même influencer l'évolution du marché ${ }^{20}$. Elle constitue la première variable discriminante dans l'étude britannique sur le sujet (Storey, 1995).

Le contact direct s'exprime, d'une part, par la vente directe à des consommateurs, à des détaillants, à des institutions ou à des entreprises manufacturières. Seulement un tiers passent par des grossistes, comme le montre le tableau 4.

TABLEAU 4

Types de clientèle

\begin{tabular}{lccccc}
\hline Types de clientèle & Consommateurs & $\begin{array}{c}\text { Entreprises } \\
\text { de détail }\end{array}$ & Grossistes & $\begin{array}{c}\text { Entreprises } \\
\text { manufacturières }\end{array}$ & Institution \\
\hline $\begin{array}{l}\text { Nombre } \\
\begin{array}{l}\text { et pourcentage } \\
\text { des entreprises }\end{array}\end{array}$ & $10(20 \%)$ & $20(41 \%)$ & $16(33 \%)$ & $29(59 \%)$ & $10(20 \%)$ \\
\hline
\end{tabular}

Ce contact peut être vu aussi par le fait que ces firmes, dans $80 \%$ des cas, le préfèrent même si elles passent également par des distributeurs, des agents ou des représentants (tableau 5).

20. Ce que nous disait pouvoir faire un producteur de machinerie pourtant beaucoup plus petit que ses principaux compétiteurs.

Revue internationale P.M.E., vol. 14, $\mathrm{n}^{\text {os }} 3-4,2001$ 
TABLEAU 5

Méthodes de commercialisation

\begin{tabular}{lcccccc}
\hline $\begin{array}{l}\text { En direct } \\
\text { de l'entreprise } \\
\text { seulement }\end{array}$ & $\begin{array}{c}\text { En direct } \\
\text { et... }\end{array}$ & $\begin{array}{c}\text { par des } \\
\text { distributeurs }\end{array}$ & $\begin{array}{c}\text { par des } \\
\text { agents } \\
\text { commerciaux }\end{array}$ & $\begin{array}{c}\text { par des } \\
\text { filiales } \\
\text { commerciales }\end{array}$ & $\begin{array}{c}\text { avec des } \\
\text { représentants }\end{array}$ & autrement \\
\hline $14(29 \%)$ & $39(80 \%)$ & $22(45 \%)$ & $17(35 \%)$ & $8(16 \%)$ & $2(4 \%)$ & $6(12 \%)$ \\
\hline
\end{tabular}

Dans le cas de clients manufacturiers, $36 \%$ des firmes font de la sous-traitance dont un peu plus de spécialité que de capacité. De même, un plus grand nombre vont recourir à la sous-traitance, dans ce cas presque autant de spécialité que de capacité.

Pour certaines entreprises, ce contact direct s'établit par des visites régulières des clients (48\%), y compris dans le cas de l'exportation, même ces visites sont alors évidemment moins fréquentes. On se sert aussi des foires, notamment pour rencontrer ces clients à l'étranger (32\%). La fréquence des visites varie entre toutes les deux semaines et une fois l'an. On utilise de même des cliniques de formation ou on fournit de l'aide technique (16\% dans les deux cas). Quatre pour cent des firmes passent par une bannière ou distribuent un journal pour garder le contact avec la clientèle.

Les liaisons particulières avec les clients peuvent être vues aussi dans le fait que $48 \%$ des dirigeants affirment se préoccuper continuellement de la qualité des produits. De plus, ces entreprises ont des pratiques de service après-vente importantes (dans $48 \%$ des firmes). Enfin, $40 \%$ traitent directement les plaintes, alors que $8 \%$ offrent un système d'assurances et de garanties.

Pour élargir leur marché, une majorité des firmes (60\%) font de la promotion écrite dans des revues spécialisées (24\%), dans des dépliants promotionnels (20\%) ainsi que dans des brochures corporatives (12\%) ou des catalogues (12\%). Vingt pour cent ont commencé à utiliser Internet pour offrir leurs services. Au total, $72 \%$ des firmes travaillent systématiquement à promouvoir la réputation de l'entreprise, soit directement (48\% par le bouche à oreille) ou par des moyens promotionnels.

Un bon nombre de ces entreprises exportent (80\% aux États-Unis), dont $41 \%$ à l'extérieur de l'Amérique du Nord et encore plus vendent aussi dans le reste du Canada ( $84 \%$ ), comme le montre le tableau 6. Au total, $96 \%$ des firmes vendent hors Québec. Le rapport français (voir l'article de Philippe Mustar dans ce numéro) accorde aussi une grande importance à l'exportation pour les PME à forte croissance françaises.

Revue internationale P.M.E., vol. 14, $\mathrm{n}^{\text {os }} 3-4,2001$ 
TABLEAU 6

Localisation des marchés

\begin{tabular}{cccccc}
\hline Régional & Québécois & Hors Québec & Reste du Canada & États-Unis & Autres pays \\
\hline $52 \%$ & $100 \%$ & $94 \%$ & $81 \%$ & $75 \%$ & $40 \%$ \\
\hline
\end{tabular}

À moyen terme, la proximité est assurée en particulier par l'innovation de produits ; $68 \%$ des entreprises en font plus ou moins systématiquement, dont une bonne partie en mettant à contribution leurs employés, déclencheurs importants d'innovation (ce qu'avaient déjà noté Burgelman et Sayles, 1986, et Cornwall et Hartman, 1988). En général, elles dépensent formellement 4,6\% de leur chiffre d'affaires en R-D, ce qui est particulièrement élevé en comparaison de la moyenne des PME, soit moins de $1 \%$ (Acs et Audretch, 1990 ; Bernard et Torre, 1994) ${ }^{21}$. L'importance de la R-D pour soutenir une forte croissance a aussi été relevée par Baldwin (1995), Ylinenpää (1997) et Liu, Tsou et Hammit (1999).

Pour soutenir cette R-D, 68 \% également assurent une veille technologique relativement formelle pour trouver des idées ou comparer leurs produits à ce qui existe et pour connaître le développement des nouvelles technologies et des nouveaux procédés ; ici aussi, l'importance de cette veille est bien supérieure à ce qui se fait en moyenne (environ $25 \%$, comme nous l'avons mesuré; Julien et al., 1999). Pour ce faire, on utilise différentes techniques dont le réseau de distributeurs.

Quant aux entreprises qui font peu ou pas de R-D, cela peut s'expliquer, du moins en partie, de diverses façons : certaines PME ont comme principaux clients d'autres entreprises, dont un bon nombre sont des donneurs d'ordres qui font eux-mêmes de la R-D et les résultats, quelle que soit l'origine ou la participation croisée, sont crédités à ces derniers; d'autres, enfin, ont comme principaux clients des consommateurs qui exigent peu de différenciation (tableau 4), comme dans le cas de la boulangerie-pâtisserie de notre échantillon.

Enfin, près de $50 \%$ des entreprises affirment contrôler plus de $40 \%$ de leur marché, comme on peut le voir au tableau 7. Cela s'explique justement par la stratégie de différenciation ou d'interstices dont parle Penrose (1959).

TABLEAU 7

Importance de l'entreprise sur son marché (34 réponses)

\begin{tabular}{ccccc}
\hline $\mathbf{3 9} \%$ et moins & Entre $\mathbf{4 0}$ et $\mathbf{4 9} \%$ & Entre 50 et 59\% & Entre 60 et 69\% & Plus de 70\% \\
\hline $18(53 \%)$ & $8(24 \%)$ & $4(12 \%)$ & $1(3 \%)$ & $3(9 \%)$ \\
\hline
\end{tabular}

21. Ou $1,5 \%$ du PIB pour toutes les firmes, quelle que soit leur taille dans les 21 pays de l'OCDE (1998). 
Encore ici, les analyses statistiques révèlent que les variables les plus discriminantes pour une forte croissance en ce qui a trait aux relations particulières avec le marché sont une communication régulière, directe et complexe avec la clientèle et l'innovation de produits.

\subsection{Des pratiques organisationnelles décentralisées et participantes}

Parmi les récentes recherches sur les entreprises à forte croissance, celles de Sexton et Seale (1997) et de Barringer, Jones et Lewis (1998) montraient que le contrôle actif sinon proactif du changement chez les entreprises à forte croissance doit nécessairement passer par une organisation complexe, décentralisée et responsable, la direction étant évidemment incapable de tout faire, à la différence de l'hommeorchestre dans les plus petites entreprises. C'est ce que nous avons trouvé dans cette étude en constatant que le plus grand nombre de ces entreprises comptaient, d'une part, un personnel cadre important qui provenait de disciplines variées et qui participait la plupart du temps aux décisions importantes et, d'autre part, des employés impliqués dans l'évolution de leurs tâches quotidiennes.

Ce troisième élément pèse plus lourd que les deux premiers. En effet, il est impossible d'avoir un bon orchestre d'improvisation si les joueurs ne se sentent pas systématiquement impliqués : ils doivent donc se percevoir comme des membres à part entière et se sentir soutenus par leur public.

Au plan de la direction ou du personnel cadre, l'organisation est complexe et décentralisée. On trouve divers spécialistes en sciences administratives (dans $98 \%$ des cas) dont dans $90 \%$ un comptable (ce qui était à prévoir), dans $20 \%$ un spécialiste en marketing et/ou en finance et $16 \%$ en ressources humaines; mais également un ou plusieurs ingénieurs (dans $63 \%$ des cas). Au total, près de $90 \%$ de ces entreprises ont au moins deux cadres spécialisés (avec des spécialités différentes) en plus de la direction de ces entreprises et $36 \%$ en ont quatre et plus. En outre, ces entreprises aident ces spécialistes à s'améliorer en leur fournissant de la formation le plus souvent de façon continue (pour $59 \%$ des entreprises dans le cas des cadres et $44 \%$ dans le cas du personnel de vente).

Ces entreprises disposent de technologies le plus souvent à jour, évitant le piège d'une technologie trop avancée par rapport à la maîtrise de celle-ci. Plusieurs d'entre elles disposent toutefois de systèmes informatisés de gestion, tels le MRP II ou le MFS. Plus de $54 \%$ d'entre elles évaluent leur technologie de production au même niveau de la concurrence mais comme un peu plus perfectionnée et efficace au plan de la gestion. Parmi ces technologies, il y a aussi l'implantation des systèmes de contrôle total de la qualité. Seulement $40 \%$ des firmes n'ont pas encore implanté un système officiel de contrôle de la qualité ; 57 \% considèrent que cette implantation les favorise par rapport à la concurrence.

Revue internationale P.M.E., vol. 14, $\mathrm{n}^{\text {os }}$ 3-4, 2001 
L'enquête révèle également que les décisions se prennent le plus souvent par consensus après discussion avec les membres de l'équipe de direction. Dans seulement $12 \%$ des cas, surtout dans les plus petites entreprises, le dirigeant prend seul les décisions importantes. Le mode de décision le plus fréquent est l'analyse rationnelle (dans $52 \%$ des cas), soit la décision après une évaluation chiffrée des avantages et des coûts. Vingt pour cent des entreprises seulement utilisent la méthode d'essai et erreur et $12 \%$, l'intuition.

Dans le cas des décisions stratégiques, 8,4 tâches sur 13 sont partagées avec les cadres, y compris un bon nombre de décisions majeures (pour $86 \%$ des entreprises). Le PDG se réserve un peu plus les tâches (en moyenne 1,9 tâche) relatives à la recherche d'occasions d'affaires et à l'analyse des menaces à la croissance de l'entreprise, ou encore délègue en bonne partie deux tâches parmi ces dernières, soit la recherche d'information technologique, la supervision du personnel, l'identification des besoins des clients ou le développement de produit. Le nombre maximal de tâches ainsi déléguées est de cinq.

Enfin, la majorité ( $80 \%)$ des entreprises pratiquent une planification stratégique flexible, soit formelle ou écrite (56\%), soit informelle ( $24 \%$ ), dont la portée se situe entre un et cinq ans. Le plan stratégique est élaboré par un comité de gestion ou de direction $(36 \%)$ et il est révisé et mis à jour de façon régulière $(52 \%)^{22}$. L'importance d'une certaine forme de planification stratégique pour mieux orienter le changement avait déjà été soulignée par Steiner et Solem (1988) et Shrader, Mulford et Blackburn (1984).

La motivation pour les cadres passe par cette participation aux décisions mais aussi par le partage des bénéfices ou par l'actionnariat. Quant aux autres employés, la participation financière relève de bonus $(52 \%)$, du partage des bénéfices $(20 \%)$ ou même de la participation à l'actionnariat $(12 \%)$. Dans $8 \%$ des cas pour les cadres et $4 \%$ pour le personnel de vente, ces derniers participent directement aux profits de l'entreprise. Au total, les trois quarts des entreprises offrent une participation financière sous une forme ou une autre à leurs cadres et à leurs employés. L'importance de cette implication financière pour soutenir la croissance a été soulignée par Sexton et Seale (1997).

Au-delà de cette participation financière, la plupart des firmes $(88 \%)$ responsabilisent leurs employés à divers niveaux. Certaines vont même jusqu'à les faire participer à des décisions de changement dans l'entreprise comme l'achat des équipements (dans $36 \%$ des cas) ou l'innovation.

22. À cela s'ajoute une planification opérationnelle (de la production). Ainsi, $84 \%$ des entreprises planifient la production au moins hebdomadairement et $52 \%$ le font à l'aide d'un programme informatique. 
Soixante-seize pour cent des dirigeants déclarent se soucier de façon importante de la communication avec le personnel, soit de façon formelle (64\%), lors de rencontres hebdomadaires ou mensuelles, soit aussi ou seulement de façon informelle $(56 \%)$. On trouve aussi divers comités qui participent à des réunions hebdomadaires avec les employés (28\% des cas). De même, certaines entreprises organisent des réunions trimestrielles ou annuelles pour l'ensemble des employés, ou encore des rencontres par département soit hebdomadaires, soit mensuelles.

De façon générale, la formation accordée au personnel (dans $88 \%$ des cas) est assurée de façon continue $(72 \%)$ ainsi qu'à l'embauche $(48 \%)$. Pour les employés, elle se retrouve le plus souvent à l'embauche $(44 \%)$ ou ponctuelle $(32 \%)$. Le personnel de bureau, pour sa part, est formé selon les besoins (48\%). Au total, le budget de formation compte pour $2,4 \%$ du chiffre d'affaires ou plus de $5 \%$ à $7 \%$ de la masse salariale ${ }^{23}$, soit beaucoup plus que la moyenne des entreprises ${ }^{24}$.

Dans $84 \%$ des entreprises, la production est planifiée d'avance, même pour la semaine $(20 \%)$ ou de façon journalière $(12 \%)$ et elle est souvent informatisée (dans $52 \%$ des cas). Le plan de production qui a pour effet d'augmenter la cohérence de l'entreprise est souvent établi par les responsables des départements de production et des ventes. Certaines entreprises produisent un rapport quotidien de production (16\% des cas), d'autres révisent le plan de production de façon régulière (12\%).

En résumé, pour l'élément organisation, les variables qui ressortent statistiquement le plus pour justifier les plus fortes croissances parmi ces entreprises (les variables discriminantes ${ }^{25}$ ) sont une grande variété de spécialités chez les cadres, l'utilisation d'un système de gestion informatisé, le recours aux technologies de dessin assisté par ordinateur, d'Internet ou d'intranet, la participation des employés à plusieurs décisions et la formation continue pour tout le personnel.

\subsection{Le recours systématique aux ressources complémentaires du milieu}

Comme dernière grande caractéristique qui répond à la septième règle de l'orchestre de jazz d'improvisation, on trouve que les gazelles recourent beaucoup plus que la moyenne aux ressources extérieures pour compléter leurs propres ressources mais aussi plus systématiquement et souvent en établissant différentes formes de

23. Si l'on considère que le coût de la masse salariale varie entre $30 \%$ et $50 \%$ du chiffre d'affaires.

24. Faute de données précises, on peut penser que l'importance de cette formation pour l'ensemble des PME ne doit pas être très loin du $1 \%$ de la masse salariale prescrit par la Loi 90 au Québec.

25. Avec une probabilité d'au moins $\mathrm{p}<0,01$.

Revue internationale P.M.E., vol. 14, nos 3-4, 2001 
partenariat. Ce recours passe d'abord par les réseaux traditionnels d'affaires, mais de façon beaucoup mieux structurée que la plupart des PME et aussi par des réseaux que Granoveter (1982) ou Woodward (1990) ont appelé des réseaux à liens faibles, moins naturels pour elles et qui vise essentiellement à favoriser ou à stimuler l'innovation (Hansen, 1999; Andriambeloson, 2000).

Dans le cas des réseaux d'affaires, au tableau 8 on relève que $36 \%$ des firmes ont conclu des accords formels avec des clients et $33 \%$, avec des fournisseurs. Cette coopération systématique touche même les concurrents dans $15 \%$ des cas pour des accords formels. De même, $26 \%$ ont établi des accords avec d'autres entreprises, dont plusieurs dans des alliances formelles très efficaces. À noter que plusieurs de ces coopérations se font avant tout pour partager de la publicité et du transport, mais aussi pour la R-D, notamment dans le partenariat avec les clients et avec d'autres entreprises. Avec les clients, ces relations permettent de développer pour eux de nouveaux produits répondant mieux à leurs besoins et qui, par la suite, peuvent être offerts à tout le marché (Peyroux, 1994); ces relations constituent des réseaux fournissant de l'information implicite particulièrement riche.

\section{TABLEAU 8}

Type de liens d'affaires avec les réseaux d'affaires et principaux objets de ces liens

\begin{tabular}{lllll}
\hline Types de liens & Avec les clients & $\begin{array}{l}\text { Avec les } \\
\text { fournisseurs }\end{array}$ & $\begin{array}{l}\text { Avec des } \\
\text { concurrents }\end{array}$ & $\begin{array}{l}\text { Avec d'autres } \\
\text { entreprises }\end{array}$ \\
\hline Partenariat formel & $36 \%$ & $33 \%$ & $15 \%$ & $26 \%$ \\
Objectifs & Publicité / & $\begin{array}{l}\text { Achats } \\
\text { de matières }\end{array}$ & $\begin{array}{l}\text { Transport / } \\
\text { distribution }\end{array}$ & $\begin{array}{l}\text { Publicité / } \\
\text { marketing } 53 \%\end{array}$ \\
les plus & marketing & premières $70 \%$ & $100 \%$ & R-D / \\
importants de & $85 \%$ & Publicité / & Publicité / & technologie $53 \%$ \\
ces accords & $\begin{array}{l}\text { Transport / } \\
\text { distribution }\end{array}$ & $\begin{array}{l}\text { marketing } \\
\text { marketing }\end{array}$ & $\begin{array}{l}\text { Matières } \\
\text { premières } 40 \%\end{array}$ \\
& $46 \%$ & $45 \%$ & $100 \%$ & Transport / \\
& R-D /technologies & & & distribution $40 \%$ \\
\hline
\end{tabular}

Les relations avec le milieu passent aussi par le recours à toutes sortes de services fournis par la région proche ou lointaine (surtout la métropole). Dans certains cas, ce recours est systématique au point que, dans une entreprise, un conseiller externe a fini par être engagé et qu'il a même fini par acheter la firme dans deux autres cas. Le tableau 9 montre que la grande majorité des firmes $(80 \%)$ recourent à plus de trois conseillers et que $49 \%$ en utilisent six et plus, ce qui est beaucoup comparé à ce qui se passe dans les PME plus traditionnelles.

Ce recours à tant de conseillers externes illustre combien ces firmes à forte croissance ont besoin de ressources complémentaires et flexibles pour faire face 
aux changements organisationnels et même opérationnels qui peuvent survenir très souvent dans leur évolution rapide et pour prendre du recul par rapport au quotidien qui accapare trop d'énergie. Ce besoin important en ressources externes pour les PME à forte croissance a été souligné par plusieurs chercheurs comme Dollinger et Kolchin (1986), Kent (1994) et Reijnders et Verjallen (1996).

Les conseillers les plus demandés sont les conseillers attachés à des firmes conseils; $82 \%$ des firmes y recourent. Viennent ensuite le banquier (63\%) suivi de près des conseillers gouvernementaux (Investissement Québec, Conseil national de recherche, Banque de développement du Canada, etc.) qui peuvent venir aussi d'un organisme régional comme le commissaire industriel. Le degré de satisfaction est relativement élevé, soit plus de 3,7 sur une échelle de 5, y compris pour les conseillers gouvernementaux. Quant aux conseillers scientifiques, nous y reviendrons plus bas.

TABLEAU 9

Nombre de spécialistes externes, type de spécialités et degré de satisfaction

\begin{tabular}{lcccccc}
\hline Nombre de conseillers & $\mathbf{0}$ & $\mathbf{1}$ à $\mathbf{3}$ & $\mathbf{4}$ & $\mathbf{5}$ & $\mathbf{6}$ à $\mathbf{8}$ & $\mathbf{9}$ et + \\
\hline N et \% d'entreprises & $4(8 \%)$ & $6(12 \%)$ & $9(18 \%)$ & $7(14 \%)$ & $14(27 \%)$ & $11(22 \%)$ \\
\hline Types de spécialités & $\begin{array}{c}\text { Firmes } \\
\text { conseil }\end{array}$ & Banquier & $\begin{array}{c}\text { Conseiller } \\
\text { gouverne- } \\
\text { mental }\end{array}$ & $\begin{array}{c}\text { Associations } \\
\text { contacts d'affaires }\end{array}$ & $\begin{array}{c}\text { Entourage } \\
\text { personnel }\end{array}$ & $\begin{array}{c}\text { Conseiller } \\
\text { scientifique }\end{array}$ \\
\hline N et \% d'entreprises & $42(82 \%)$ & $32(63 \%)$ & $31(61 \%)$ & $16(31 \%)$ & $13(25 \%)$ & $21(41 \%)$ \\
\hline Degré de satisfaction* & 4,04 & 3,9 & 3,8 & 3,7 & 4,2 & 4 \\
\hline
\end{tabular}

Échelle: 1 : pas satisfait ; 2 : peu satisfait ; 3 : satisfait ; $4:$ assez satisfait $; 5:$ très satisfait.

Ces entreprises utilisent plus souvent que leurs consœurs l'aide gouvernementale, notamment pour soutenir la R-D (83\% des entreprises), pour aider l'exportation (45\%) et pour la formation du personnel (43\%) et de la direction (19\%).

Mais l'information la plus riche, notamment pour soutenir l'innovation, passe par la présence de conseillers scientifiques (comme l'indique le tableau 9) et par des relations avec les institutions d'enseignement de troisième cycle ou les centres de recherche, soit des réseaux à liens faibles, fournissant le plus souvent des signaux faibles $^{26}$, comme nous l'avons mentionné. Au tableau 10, on voit que ces relations

26. Les signaux faibles sont ceux que généralement les gens d'affaires perçoivent mal, soit parce qu'ils s'y intéressent peu, soit à cause d'un langage, d'objectifs et de formes organisationnelles trop éloignées de leurs préoccupations ou de leurs façons d'appréhender la réalité.

Revue internationale P.M.E., vol. 14, $\mathrm{n}^{\text {os }} 3-4,2001$ 
sont relativement importantes : $19 \%$ des entreprises ont des accords formels dans le premier cas et $13 \%$ dans le second cas, ce qui est beaucoup par rapport à ce qui se passe traditionnellement, alors que ces accords sont peu répandus (OCDE, 1993). Comme cela était à prévoir, ces relations portent sur la formation du personnel et sur la recherche et développement pour soutenir l'effort important en innovation.

TABLEAU 10

Type de liens d'affaires avec des réseaux à signaux faibles et objets de ces liens

\begin{tabular}{lcc}
\hline Types de liens & $\begin{array}{c}\text { Avec des institutions } \\
\text { d'enseignement }\end{array}$ & $\begin{array}{c}\text { Avec des centres } \\
\text { de recherche }\end{array}$ \\
\hline $\begin{array}{c}\text { Partenariat formel } \\
\text { (maillage ou coopération) }\end{array}$ & $19 \%$ & $13 \%$ \\
$\begin{array}{l}\text { Objectifs partagés } \\
\text { les plus importants }\end{array}$ & $\begin{array}{c}\text { Formation de personnel } 67 \% ; \\
\text { R-D/techno. } 33 \%\end{array}$ & $\begin{array}{c}\text { R-D/techno. } 63 \% ; \\
\text { production } 27 \%\end{array}$ \\
\hline
\end{tabular}

Quant aux relations avec le milieu, la dernière variable, le partenariat avec des institutions d'enseignement et des centres de recherche constitue statistiquement parlant une des variables corrélées avec une plus forte croissance, en plus $d u$ recours à l'aide gouvernementale.

\section{Les variables les plus discriminantes}

Nous avons effectué une série d'analyse en séparant les deux principaux types de trajectoires de croissance, soit une croissance continue plus ou moins rapide et une croissance accélérée ou très accélérée.

Comme on peut le vérifier au tableau 11, les variables qui, statistiquement, différencient ces deux trajectoires sont les suivantes: 1) le type de technologies privilégié, l'entreprise utilisant plus fortement la CAO dans le cas d'une croissance en continu tandis qu'elle recourt plus à des systèmes informatisés de la production dans celui d'une croissance accélérée ; 2) la motivation de l'entrepreneur, dans le premier cas pour assurer la relève et dans le second pour bâtir son entreprise; 3) la qualité des composantes en tant que sous-traitant de capacité ou de spécialité, dans le premier cas, et des relations plus directes avec les clients, dans le deuxième cas ; 4) une veille technologique réalisée à l'interne dans le premier cas, alors que dans le second, on recourt à de l'aide extérieure pour ce faire et on travaille avec des centres de recherche. 
TABLEAU 11

Variables différenciant le mieux

les deux grandes trajectoires de forte croissance

\begin{tabular}{|c|c|c|}
\hline Variables & $\begin{array}{l}\text { Croissance continue } \\
\text { ( } 24 \text { entreprises) }\end{array}$ & $\begin{array}{l}\text { Croissance accélérée } \\
\text { (16 entreprises) }\end{array}$ \\
\hline $\begin{array}{l}\text { L'organisation } \\
\text { Une des technologiques privilégiées }\end{array}$ & $\mathrm{CAO}$ & Contrôle informatisé de la production \\
\hline $\begin{array}{l}\text { L'entrepreneur } \\
\text { Caractéristiques du dirigeant } \\
\text { Motivation particulière }\end{array}$ & $\begin{array}{l}\text { A participé à la création } \\
\text { de l'entreprise. } \\
\text { Réussir la relève. }\end{array}$ & Bâtir son entreprise \\
\hline $\begin{array}{l}\text { Les relations avec le marché } \\
\text { Éléments particuliers de vente } \\
\text { Spécialisation }\end{array}$ & $\begin{array}{l}\text { La qualité des composantes } \\
\text { La satisfaction des consommateurs } \\
\text { La sous-traitance de capacité et de } \\
\text { spécialité }\end{array}$ & Des relations directes avec la clientèle \\
\hline $\begin{array}{l}\text { Les relations } \\
\text { avec l'environnement } \\
\text { La veille technologique }\end{array}$ & $\begin{array}{l}\text { L'entreprise effectue } \\
\text { elle-même la veille. }\end{array}$ & $\begin{array}{l}\text { Utilisation des ressources extérieures } \\
\text { pour la veille } \\
\text { Des relations avec des centres de } \\
\text { recherche } \\
\text { Recours à des conseils externes } \\
\text { pour la formation }\end{array}$ \\
\hline
\end{tabular}

Nous avons aussi réalisé quelques analyses typologiques (à partir de 42 entreprises dont on a pu obtenir toute l'information $)^{27}$ qui indiquent que les PME à plus forte croissance sont plus récentes (65\% des firmes ont moins de 15 ans) et sont dirigées par des entrepreneurs plutôt jeunes (moins de 40 ans), spécialisés, mieux formés et avides de formation continue, ce qu'ont aussi montré Liu, Tsou et Hammitt (1999); de plus, ils s'entourent de cadres de leur âge avec qui ils partagent la plus grande partie des tâches (tableau 12).

En contrepartie, dans les firmes plus anciennes, le dirigeant est âgé et moins spécialisé, mais a plus d'expérience dans le secteur; il dirige sa firme depuis plus longtemps et était probablement présent au moment de sa création ; il a suivi moins de formation particulière au cours des cinq dernières années; de même, son équipe de direction est âgée et il lui délègue un plus grand nombre de tâches.

27. Cette analyse typologique peut être discutable compte tenu du peu d'entreprises dans l'échantillon. Toutefois, les perceptions qualitatives des entrevues confirment les résultats trouvés.

Revue internationale P.M.E., vol. 14, nos 3-4, 2001 
TABLEAU 12

Analyse typologique des liens

entre certaines caractéristiques de la direction et le partage des tâches et une croissance moins forte ou plus forte (42 entreprises)

\begin{tabular}{|c|c|c|c|}
\hline & Moins forte & Plus forte & \\
\hline Variables & 15 entreprises & 27 entreprises & $\begin{array}{l}42 \text { entreprises } \\
\text { en moyenne }\end{array}$ \\
\hline Âge du dirigeant & plus âgé & plus jeune & \\
\hline Ancienneté à la direction & plus ancienne & plus récente & \\
\hline Origine de la firme & généralement le fondateur & diverse & \\
\hline $\begin{array}{l}\text { Années d'expérience } \\
\text { dans le secteur }\end{array}$ & plusieurs années & récentes & \\
\hline Âge des cadres & âgés & plutôt jeunes & \\
\hline $\begin{array}{l}\text { Formation de base } \\
\text { spécialisée } \\
\text { de l'entrepreneur }\end{array}$ & spécialisée à $64 \%$ & spécialisée à 100 \% & spécialisée à $88 \%$ \\
\hline $\begin{array}{l}\text { Formation continue } \\
\text { pour l'entrepreneur }\end{array}$ & $14 \%$ en suivent & $100 \%$ en suivent & $69 \%$ en suivent \\
\hline $\begin{array}{l}\text { Tâches effectuées } \\
\text { en collaboration }\end{array}$ & 6,8 tâches & 9,1 tâches & 8,4 tâches \\
\hline Tâches déléguées & 3,7 tâches & 1,1 tâches & 2,0 tâches \\
\hline
\end{tabular}

\section{Conclusion}

Les 17 variables qui distinguent les PME à plus forte croissance des autres peuvent se résumer par les quatre variables suivantes:

1. Une organisation complexe, décentralisée et participative;

2. Une orientation dynamique et cohérente donnée par la direction;

3. Une différenciation basée sur l'innovation et l'échange de savoirs avec les clients ;

4. Un savoir enrichi par de la formation continue et des liens privilégiés avec des réseaux à signaux forts et à signaux faibles dans l'environnement.

Ces variables sont relevées dans le travail précurseur d'Édith Penrose qui soutenait déjà en 1959 que ce qui freinait la croissance des firmes, c'était la capacité même de l'organisation à gérer cette croissance et à contrôler l'évolution de sa structure (voir Barth, 1999, et Zack, 2000). Autrement dit, les PME à forte croissance sont capables de contrôler et de se réaménager systématiquement en comptant sur les cadres et les employés clés (les joueurs) qui se repositionnent dans l'organisation (l'orchestre) et utilisent ou inventent diverses techniques ou méthodes (leurs 
instruments) pour analyser la situation changeante et pour prendre les décisions appropriées rapidement (changeant de rythme $)^{28}$, quitte à changer de rôle momentanément. Pendant ce temps, la direction accepte certaines erreurs ${ }^{29}$ et les changements imprévus dans l'organisation pour mieux déceler les occasions d'affaires et se consacrer aux grands défis que pose la concurrence.

De plus, l'efficacité de l'organisation passe par le recours à des conseillers externes (des joueurs visiteurs) pour compléter le travail des cadres et pour les assister dans de nouveaux projets (pour trouver de nouvelles formes musicales). Ce recours marque justement la capacité de ces organisations à évoluer avec l'apport extérieur, en plus de la formation continue. En particulier, comme l'innovation est une clé pour soutenir le développement du marché et pour répondre continuellement à l'évolution des besoins de la clientèle, l'aide de conseillers scientifiques et les relations avec des centres de recherche sont importantes. La relation entre la complexité de l'organisation et l'innovation est cohérente avec les études sur les variables favorisant cette dernière, comme l'ont montré Cohn et Turyn (1980). On peut penser que cette relation est logique étant donné que l'innovation est essentiellement un processus de contradictions (de désordre) et de changement comme l'est d'une manière plus globale la forte croissance.

En d'autres mots, les gazelles appliqueraient le principe de la variété requise en analyse systémique ${ }^{30}$. En effet, comme elles doivent évoluer rapidement pour faire face aux changements rapides qui s'opèrent dans leur marché, tant en nombre (augmentation rapide des commandes et, souvent, du nombre de clients) qu'en qualité (des réponses évoluant rapidement selon les besoins et par l'innovation), elles doivent systématiquement se reconfigurer en variant et en augmentant la qualité et le nombre de ressources, notamment en recourant à des ressources externes. Les ressources internes, étant décentralisées et participatives, peuvent répondre à différentes demandes sans que la direction soit obligée de voir à tout, ce qu'elle ne pourrait faire de toute façon compte tenu du nombre d'ajustements à faire. Les ressources externes, essentielles par ailleurs, n'étant pas dans le feu de l'action, servent à prendre

28. Règles 3 et 4 du jazz d'improvisation.

29. Barret (1998) soutient que les erreurs peuvent servir d'étincelles pour envisager de nouvelles orientations, ce que conteste Zack (2000), bien que cela puisse très bien fonctionner dans le jazz post-bebop.

30. Ce principe explique qu'il faut qu'une organisation soit aussi complexe que le système dans lequel il interagit s'il veut interagir avec lui. Par exemple, Mélèse (1979) a montré qu'une organisation fortement hiérarchique ne peut évoluer que lentement et qu'il faut à ce moment-là qu'elle contrôle son environnement par diverses mesures monopolistiques : «Une hiérarchie formelle dans laquelle toute la variété provient du sommet n'est qu'un dispositif de démultiplication qui ne présente aucun caractère de capacité de contrôle, d'adaptation au d'apprentissage » (p. 73).

Revue internationale P.M.E., vol. 14, $\mathrm{n}^{\text {os }} 3-4,2001$ 
du recul pour mieux adapter l'organisation aux nouveaux besoins (Donckel et Lambrecht, 1995). Enfin, la direction a avant tout comme mandat de donner de la cohérence à tout cela et, par conséquent, de protéger l'harmonie (dans le sens musical du terme). C'est donc une organisation capable d'absorber rapidement les impulsions du marché (les informations données implicitement par les auditeurs), en $s^{\prime}$ adaptant systématiquement pour y répondre le mieux et le plus rapidement possible.

En ce sens, les PME à forte croissance sont des organisations dans le sens propre du mot, soit des organismes vivants ou des systèmes ouverts à base d'une culture de changement (Hofstede, 1994) qui s'auto-adaptent en s'ajustant systématiquement à mesure qu'elles participent à l'évolution de leur marché, par un processus de clôture opérationnelle ${ }^{31}$, soit la capacité d'une organisation à intervenir dans le changement en changeant elle-même à mesure qu'elle intervient selon un mouvement en double boucle (comme l'expliquent Argyris et Schön (1978) à propos de l'apprentissage) ou encore en spirale.

Bref, ces entreprises ont une capacité particulière de gérer l'improvisation dictée par le changement systématique pour satisfaire les besoins changeants de leur clientèle. Ainsi, ces entreprises ne peuvent évoluer ${ }^{32}$ qu'à la condition que leur structure évolue continuellement en changeant même de registre (passant de petite à moyenne et même à grande), comme c'est le cas du jazz qui est passé du New Orleans (1890 à 1920) au post-bebop (1950...), soit le style qui favorise le mieux le jazz d'improvisation, car il permet de varier le rythme au cours de la performance (Zack, 2000). L'improvisation accompagnée d'inattendu n'est-elle pas l'essence même du grand jazz?

31. Nous avions utilisé auparavant le mot autopoïese comme le fait Vagaggini (1991), mais il semble que cette analogie tirée de la biologie moléculaire ne convienne pas lorsqu'on parle de système organisationnel, comme l'explique Verstraete (1999) qui propose d'utiliser plutôt la notion de clôture organisationnelle par lequel le changement rapide est contrôlé par la volonté d'autoconservation du système. Voir aussi à ce propos Le Moigne (1977).

32. La forte croissance n'est pas toujours continue sur une longue période, comme on l'observe chez les firmes de notre échantillon qui ont connu des évolutions sporadiques ou des changements de rythme. Baldwin (1995) a relevé d'ailleurs que près de $50 \%$ des PME à forte croissance ne survivent pas après dix ans. 


\section{AnNeXe I}

Les entreprises étudiées avec leur taux de variation de 1990 à 1998 quant à l'emploi et le chiffre d'affaires

\begin{tabular}{|c|c|c|c|c|c|}
\hline $\begin{array}{l}\text { Numéro } \\
\text { de } \\
\text { l'entreprise }\end{array}$ & Principaux produits & $\begin{array}{l}\text { Variation } \\
\text { annuelle } \\
\text { des } \\
\text { emplois } \\
\text { (en \%) }\end{array}$ & $\begin{array}{l}\text { Variation } \\
\text { annuelle } \\
\text { du } \\
\mathrm{CA} \\
(\text { en \%) }\end{array}$ & $\begin{array}{l}\text { Type de } \\
\text { trajectoire }\end{array}$ & Observations \\
\hline $46^{1}$ & Services comptables & 341,6 & $\ldots$ & accélérée & accélération à partir de 1993 \\
\hline 62 & Développ. logiciels & 112,5 & 67,7 & continue lente & $\begin{array}{l}\text { saut important en } 1996 \\
\text { (emploi) }\end{array}$ \\
\hline $64(5 \text { ans })^{2}$ & Services audiovisuels & 112,5 & 37,5 & continue & $\begin{array}{l}\text { saut en } 1995(\mathrm{CA}) \\
\text { et } 1996 \text { (emploi) }\end{array}$ \\
\hline $25^{2}$ & Services informatiques & 93,7 & $-3,5$ & $\begin{array}{l}\text { accélérée } \\
\text { (emploi) }\end{array}$ & décroissance du CA \\
\hline 34 (4 ans) & Machinerie pour le meuble & 92,0 & 146,0 & très accélérée & surtout après 1995 \\
\hline 17 & Produits de communication & 87,5 & 112,5 & continue & fusion en 1996 \\
\hline 45 & Produits électroniques & 81,3 & $\ldots$ & continue lente & très régulières \\
\hline 54 & $\begin{array}{l}\text { Produits structures } \\
\text { métalliques }\end{array}$ & 78,4 & 67,0 & accélérée & $\begin{array}{l}\text { irrégulière, ralentissement } \\
\text { après } 1993\end{array}$ \\
\hline 6 & Matériel de transport & 77,0 & 140,0 & très accélérée & ralentissement après 1995 \\
\hline 11 & Meubles institutionnels & 70,9 & 70,9 & très accélérée & surtout après 1993 \\
\hline 26 & Matériel de transport & 62,5 & $\ldots$ & $\ldots$ & $\ldots$ \\
\hline 21 & Remorques commerciales & $\ldots$ & 54,3 & $\ldots$ & $\cdots$ \\
\hline 5 & Produits de plastique & 51,4 & 12,5 & très accélérée & $\begin{array}{l}\text { retournement après } 1997 \\
\text { (emploi) }\end{array}$ \\
\hline $36^{3}$ & Bureau d'ingénieurs & 47,9 & 83,6 & continue lente & $\begin{array}{l}\text { le CA a fléchi un moment } \\
\text { en } 1996\end{array}$ \\
\hline 28 & Produits de plastique & 41,0 & 37,5 & $\ldots$ & - \\
\hline 47 & Équipements de sécurité & 40,9 & 24,7 & très accéléré & $\ldots$ \\
\hline 49 & Produits pharmaceutiques & 39,6 & $\ldots$ & très accéléré & surtout après 1992 \\
\hline 23 & Produits électroniques & 37,5 & $\ldots$ & $\ldots$ & $\ldots$ \\
\hline 66 & Vêtements professionnels & 35,6 & 40,2 & continue rapide & accélération en 1995 \\
\hline 50 & $\begin{array}{l}\text { Moules et matrices } \\
\text { métalliques }\end{array}$ & 34,4 & $\cdots$ & accélérée & $\begin{array}{l}\text { de } 1993 \text {, mais chute } \\
\text { entre } 1994 \text { et } 1996\end{array}$ \\
\hline 13 & Industrie de ressorts & 33,4 & 26,0 & continue rapide & un peu irrégulière \\
\hline 9 & Produits en caoutchouc & 30,0 & 40,6 & accélérée & après 1993 \\
\hline 14 & Boulangerie/pâtisserie & 30,0 & 28,6 & très accélérée & à partir de 1992 (emploi) \\
\hline 48 & Peintures et vernis & 28,6 & $\cdots$ & continue lente & léger pic en 1992 \\
\hline 3 & Panneaux de particules & 28,1 & 31,2 & accélérée & $\begin{array}{l}\text { accélération après } 1993 \\
\text { (emploi) }\end{array}$ \\
\hline 8 & Jouets et jeux & 28,1 & 58,4 & continue rapide & retournement en 1995-1996 \\
\hline 10 & $\begin{array}{l}\text { Portes et fenêtres } \\
\text { métalliques }\end{array}$ & 26,6 & 29,6 & très accélérée & saut entre 1992 et 1993 \\
\hline
\end{tabular}

Revue internationale P.M.E., vol. 14, $\mathrm{n}^{\text {os }} 3-4,2001$ 


\section{AnNeXe I (suite)}

Les entreprises étudiées avec leur taux de variation de 1990 à 1998 quant à l'emploi et le chiffre d'affaires

\begin{tabular}{|c|c|c|c|c|c|}
\hline $\begin{array}{l}\text { Numéro } \\
\text { de } \\
\text { l'entreprise }\end{array}$ & Principaux produits & $\begin{array}{l}\text { Variation } \\
\text { annuelle } \\
\text { des } \\
\text { emplois } \\
\text { (en \%) }\end{array}$ & $\begin{array}{l}\text { Variation } \\
\text { annuelle } \\
\text { du } \\
\text { CA } \\
(\text { en \%) }\end{array}$ & $\begin{array}{l}\text { Type de } \\
\text { trajectoire }\end{array}$ & Observations \\
\hline 59 & Produits alimentaires & 26,4 & 20,3 & $\begin{array}{l}\text { très accélérée } \\
(\mathrm{em} .)\end{array}$ & le CA plus stable \\
\hline 16 & Articles d'ameublement & 25,4 & 37,4 & continue & saut entre 1992 et 1993 \\
\hline 33 & Machinerie pour scieries & 21,6 & 115,8 & continue lente & quelque peu irrégulière \\
\hline 4 & Machinerie pour scieries & 21,0 & 42,5 & continue rapide & accélération à partir de 1995 \\
\hline 29 & Tourbières & 20,0 & 41,6 & $\ldots$ & \\
\hline 43 & $\begin{array}{l}\text { Moules et matrices } \\
\text { métalliques }\end{array}$ & 18,6 & 29,8 & irrégulière & $\begin{array}{l}\text { chute entre } 1988 \text { et } 1994, \\
\text { retournement 1994-1997 }\end{array}$ \\
\hline $7^{4}$ & Camionnage & 16,0 & 25,0 & continue & légère accélération en 1993 \\
\hline 1 & Instrument aratoire & 14,2 & 13,5 & continue lente & légère accélération en 1994 \\
\hline 12 & Meubles de maison en bois & 14,1 & 44,7 & continue rapide & accélération en 1994 \\
\hline 24 & Scierie et usine de bardeau & 9,6 & 44,2 & $\ldots$ & $\ldots$ \\
\hline $27^{1}$ & Bureau de comptable & 9,6 & 15,8 & $\ldots$ & $\ldots$ \\
\hline 15 & Huiles-graisses lubrifiantes & 9,4 & 25,0 & continue & ralentissement après 1995 \\
\hline 61 & Produits de plastique & 7,9 & 5,9 & continue & $\begin{array}{l}\text { saut en } 1992 \\
\text { et ralentissement en } 1995\end{array}$ \\
\hline 35 & Atelier d'usinage & 8,4 & 15,2 & continue lente & $\begin{array}{l}\text { accélération entre } 1994 \\
\text { et } 1996\end{array}$ \\
\hline 51 & Enseignes et étalages & 7,5 & 19,5 & continue lente & pic en 1993 \\
\hline 56 & Produits en verre & 6,7 & $\ldots$ & continue & recul en 1995 \\
\hline 63 & $\begin{array}{l}\text { Pellicules-feuilles } \\
\text { en plastique }\end{array}$ & 2,7 & 10,9 & $\begin{array}{l}\text { continue } \\
\text { (emploi) }\end{array}$ & CA stable sauf en 1997 \\
\hline 57 & Produits pharmaceutiques & 5,3 & 14,6 & continue & $\begin{array}{l}\text { accélération entre } 1983 \\
\text { et } 1988\end{array}$ \\
\hline 2 & Machinerie et équipements & 4,6 & 18,7 & continue & petit saut en $1996(\mathrm{CA})$ \\
\hline 65 & Produits alimentaires & 4,3 & 57,6 & accélérée & chute en 1992 \\
\hline 58 & Fibres-filaments chimiques & 1,6 & 19,1 & irrégulière & $\begin{array}{l}\text { le CA fait un bond } \\
\text { de } 1995 \text { à } 1998\end{array}$ \\
\hline 22 & Armoires et placards & 0,4 & 6,7 & continue lente & $\begin{array}{l}\text { chute en 1992, } \\
\text { accélération du CA en } 1993\end{array}$ \\
\hline $37^{2}$ & Bureau d'ingénieurs & 0,0 & 23,6 & irrégulière & $\begin{array}{l}\text { fusion en 1996, chute } \\
\text { en } 1997\end{array}$ \\
\hline 32 & Produits en verre & 0,0 & 19,5 & irrégulière & saut momentané en 1997 \\
\hline 30 & Matériel de transport & $\ldots$ & $\ldots$ & $\ldots$ & $\ldots$ \\
\hline
\end{tabular}

$\ldots$ : non disponible; 1 : entreprise de comptabilité et de tenue de livres $; 2:$ informatique $; 3:$ firmes d'ingénieurs ; 4 : firme de camionnage. 


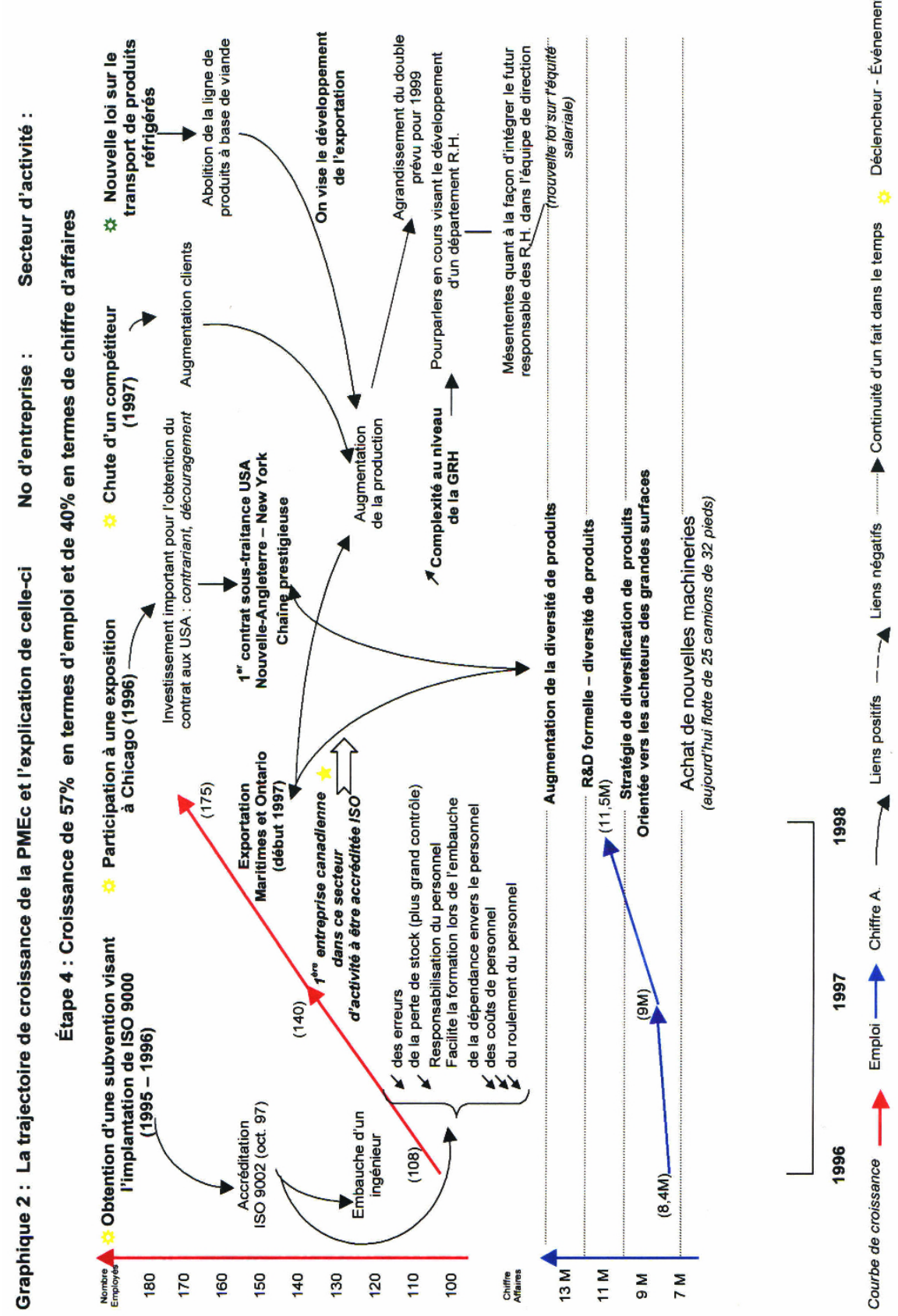

Revue internationale P.M.E., vol. 14, nºs 3-4, 2001 


\section{Bibliographie}

ACs, Z. et D. Audretsch (1990), Innovation and Small Firms, Cambridge, MIT Press.

ANDRIAMBELOSON, E. (2000), «Innovation dans les PME manufacturières et relations avec des réseaux à signaux faibles », Mémoire de maîtrise, Institut de recherche sur les PME, Université du Québec à Trois-Rivières.

ARGYRIs, C. et D. SCHÖN (1978), Organizational Learning, Don Mills, Addison-Wesley.

ARIAN, E. (1971), Bach, Beethoven and Bureaucraty, Alabama, University of Alabama Press, cité par Weick et al. (1973).

AVENIER, M.J. (dir.) (2000), Ingénierie des pratiques collectives. La cordée et le quatuor, Paris, L'Harmattan, particulièrement le chapitre 5.

BALDWIN, J.R. (1995), «L'innovation, la clé de la réussite des petites entreprises », Document de recherche $n^{\circ} 76$, Direction des études statistiques, Ottawa, Statistique Canada, février.

BALDWIn, J.R., C. CHANDlER et T. PAPAILIADIS (1994), Stratégies pour le succès. Le profil des PME en croissance au Canada, Ottawa, Statistique Canada, $\mathrm{n}^{\circ}$ 61-523ER.

BARRET, F.J. (1998), « Creativity and improvisation in jazz and organizations : implications for organizational learning », Organization Science, numéro special sur la métaphore du jazz et l'organisation, vol. 9, nº 5, p. 605-622.

BARRETT, F.J. et K. PEPLOWSKI (1998) «Minimal structure within a song : an analysis of "All of Me" », Organization Science, vol. 9, n 5, p. 543-623.

BARRINGER, B.R., F.F. JONES et P.S. LEWIS (1998), « A qualitative study of the management practices of rapid-growth firms and how rapid-growth firms mistigate the managerial capacity problem », Journal of Developmental Entrepreneurship, vol. 3, no 2, p. 97-140.

BARTH, H. (1999), «Barriers to growth in small firms », Mémoire de licence, Université de Luleå.

BERLINER, P.F. (1994), Thinking in Jazz : The Infinite Art of Improvisation, Chicago, University of Chicago Press.

BERnARD, J. et A. TORRE (1994), «La dynamique d'innovation de R-D des PMI françaises », Revue internationale PME, vol. 7, $\mathrm{n}^{\text {os }} 3-4$, p. 29-41.

Birch, D., A. Haggerty, et W. PARsons (1997), Corporate Almanac, Cambridge, Cognetic Inc.

BRown, S.L. et K.M. EISENHARDT (1998), Competitive on the Edge. Strategy as Structured Chaos, Boston, Harvard Business School Press, chap. 2.

Burgelman, R. et L. SAYLES (1986), Inside Corporate Venturing, New York, Free Press.

Chanlat, A. et M. Dufour. (dir.) (1985), La rupture entre l'entreprise et les hommes. Le point de vue des sciences de la vie, Montréal, Québec-Amérique. 
CHoo, C.W. (1998), The Knowing Organization. How Organization Use Information to Construct Meaning, Create Knowledge and Make Decisions, New York, Oxford University Press, p. 6 et 7.

COHN, S.F. et R.M. TURYN (1980), « The structure of the firm and the adoption of progress innovations », IEEE Transactions on Engineering Management, EM-27, n ${ }^{\circ} 4$, p. $98-102$.

CORNWALl, J.P. et F.A. HARTMAN (1988), « A model of organizational entrepreneurship », dans Actes du Congrès international de l'ICSB, Boston, p. 63-67.

DAVIDSSON, P. (1989), «Entrepreneurship and after? A study of growth willingness in small firms », Journal of Business Venturing, vol. 4, no 3, p. 211-226.

Deloitte \& Touche Management Solutions (1999), Growth Builders, Ministère du Développement économique et du Commerce de l'Ontario, septembre.

DivaY, M. (1999), «Comportement de croissance des entreprises de taille moyenne», Revue Gestion 2000, nº 6, nov.-déc., p. 59-80.

Dollinger, M. et M. Kolchin (1986), « Purchasing and the small business », American Journal of Small Business, vol. 10, no 3, p. 33-45.

DonCKELs, R. et J. LAMBRECHT (1995), « Networks and small business growth: an explanatory model », Small Business Economics, vol. 7, nº 3, p. 273-289.

DYKE, L., E. FISHER et A. R. REUBER (1992), «Inter-industry examination : the impact of owner experience firm on performance », Journal of Small Business Management, vol. $30, \mathrm{n}^{\circ} 4$, p. $72-87$.

GRANOVETER, M. (1982), « The strength of weak ties : a network theory revisited », dans P.V. Marsden et N. Lin (dir.), Social Structure and Network Analysis, Beverly Hills, Sage, p. 105-130.

HAMBRICK, D.C. et L.M. CROIZER (1985), « Stumblers and stars in the management of rapid growth », Journal of Business Venturing, vol. 1, $\mathrm{n}^{\circ}$ 1, p. 31-45.

HANSEN, M.T. (1999), «The search-transfer problem: the role of weak ties in sharing knowledge across organization subunits », Administrative Science Quarterly, vol. $44, n^{\circ} 1$, p. 82-111.

HATCH, M.J. (1997), « Exploring the empty space of organization », Cahier de recherche, Cranfield, Angleterre, Cranfield School of Management.

Hofstede, G. (1994), Culture and Organizations : The Software of the Mind, New York, Harper and Collins.

Huberman, A.B. et B.M. Miles (1991), Analyse des données qualitatives, Bruxelles, De Boeck.

JACOB, R., P.-A. JULIEN et L. RAYMOND (1997), « Competitivity, knowledge and collective learning in network firm », VII ${ }^{e}$ Forum international sur le management technologique, Kyoto, Japon, 3-7 nov.

JULIEN, P.-A. (2000), L'entrepreneuriat au Québec de 1980 à 2005, Montréal, Éditions Transcontinental; Québec, Éditions Entreprendre.

Revue internationale P.M.E., vol. 14, $\mathrm{n}^{\text {os }} 3-4,2001$ 
Julien, P.-A., L. Raymond, R. Jacob et C. Ramangalahy (1999), «Types of technological scanning in manufacturing SMEs : an empirical analysis of patterns and determinants », Entrepreneurship and Regional Development, vol. 11, n 2.

Julien, P.-A. et M. MARCHESNAY (1997), L'entrepreneuriat, Paris, Economica.

KENT, P. (1994), «Management advisory services and the financial performance of clients », International Small Business Journal, vol. 12, n 4, p. 45-58.

KIM, W.C. et R. MAUGorgne (1997), «Value innovation: the strategic logic of high growth », Harvard Business Review, jan.-févr.

Le Moigne, J.L. (1977), La théorie du système général, Paris, Presses universitaires de France, notamment p. 187-188.

LiU, J.T., M.W. Tsou et J.K. HAMmiTt (1999), « Do small plants grow faster? Evidence from the Taiwan electronic industry », Economic Letters, vol. 85, nº 2, p. 121-129.

MCPHEE, R. (1990), «Alternative approaches to integrating longitudinal case studies », Organization Science, vol. 1, no 4, p. 393-405.

MÉLÈSE, J. (1979), Approches systémiques des organisataions. Vers l' entreprise à complexité humaine, Paris, Anthropos.

MinguZZI, A. et R. PASSARO (2000), «The network of relationships between the economic environment and the entrepreneurial culture in small firms », Journal of Business Venturing, vol. 16, n ${ }^{\circ}$ 2, p. 181-216.

MitRA, R. et V. PINGALI (1999), «Analysis of growth stages in small firms : a case study of automobile ancillaries in India », Journal of Small Business Management, vol. 37,

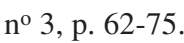

MORSE, J. (1994), « Designed founded qualitative research », dans Handbook of Qualitative Research, Londres, Sage Publications.

OCDE (1993), Les petites et moyennes entreprises : technologie et compétitivité, Paris.

OCDE (1998), Principaux indicateurs de la science et de la technologie, Paris.

PENROSE, E.T. (1959), Facteurs, conditions et mécanismes de la croissance des entreprises (traduction de son ouvrage par S.L. Mayret, Paris, Les Éditions Hommes et Techniques, 1963).

PeyrouX, C. (1994), «Produits robotiques et partenariat: les besoins des PME», dans P.-A. Julien (dir.), Pour des PME de classe mondiale, Montréal, Éditions Transcontinental, p. 199-214.

REIJNDERS, W.J.M. et M.M. VERJALLEN (1996), « Strategic alliance among small retailing firms : empirical evidence for the Netherlands », Journal of Small Business Management, vol. $34, \mathrm{n}^{\circ} 1$, p. 36-45.

RICEUR, P. (1975), La métaphore vive, Paris, Seuil.

Sabatella, M. (1992), A Whole Approach to Jazz Improvisation, Lawndale, ADG Publications. 
SANDBERG, W.R. et C.W. HOFFER (1987), «Improving new venture performance : the role of strategy, industry structure and the entrepreneur », Journal of Business Venturing, vol. $2, \mathrm{n}^{\circ} 1$, p. $5-22$.

SeXton, D.L. et F.I. SEALE (1997), Leading Practices of Fast Growth Entrepreneurs, Kansas City, National Center for Entrepreneurship Research at the Kauffman Foundation.

SCHMitT, C. (1999), La dynamique de la valeur : contribution à la création de valeur en P.M.E. par la notion de désordre, Thèse de doctorat, Institut national polytechnique de Lorraine, 10 décembre.

SiEgel, R., E. Siegel et I.C. MACMillan (1993), « Characteristics distinguishing highgrowth ventures », Journal of Business Venturing, vol. 9, n 2, p. 169-180.

Shrader, C.B., C.L. Mulford et V.L. BlackBuRN (1984), «Strategic and operational planning, uncertainty and performance in small firms », Journal of Small Business Management, vol. 27, $\mathrm{n}^{\mathrm{o}}$ 4, p. 45-60.

STEINER, M. et O. SOLEM (1988), «Factors for success in small manufacturing firms », Journal of Small Business Management, vol. 26, $\mathrm{n}^{\circ}$ 1, p. 51-56.

STOREY, D. (1995), The Ten Percenters, Rapport préparé pour la firme Andersen \& Cie, Londres.

VAGagGini, V. (1991), «Quattro paradigmi per un distretto », dans S. Conti et P.-A. Julien (dir.), Miti e realità del modello italiano. Letture sulle' economia periferica, Bologne, Pàtron Editore, p. 187-212.

Verstraete, T. (1999), Autopsie et sciences de gestion: excès d'éclectisme, Cahier de recherche du Centre lillois d'analyse et de recherche sur l'évolution des entreprises, IAE de Lille.

YIN, R.K. (1989), Case Study Research. Design and Method, Beverley Hills, Sage Foundation.

WeICK, K.E., D.P. GILFILlan et T.A. KEITH (1973), « The effect of composer credibility on orchestra performance », Sociometry, sept.

YLINENPÄÄ, H. (1997), Managing Competence Development and Acquisition in Small Manufacturing Firms, Thèse de doctorat, Université de Luleå.

WOLFE, J. et C. JACKSON (1987), « Creating models of the strategic decision-making process via participants recalls : a free simulation examination », Journal of Management, vol. $13, \mathrm{n}^{\mathrm{o}} 1$, p. 123-134.

WoOdward, W.J. (1990), A Social Theory of Entrepreneurship : An Empirical Studies, Thèse de doctorat, site Internet UMI.

ZACK, M.H. (2000), «Jazz improvisation and organizing: once more from the top », Organization Science, vol. 11, n 2, p. 227-234.

Revue internationale P.M.E., vol. 14, $\mathrm{n}^{\mathrm{os}}$ 3-4, 2001 